\title{
LA COMUNICACIÓN COMO FACTOR CLAVE EN LA IMPLANTACIÓN DE LA RESPONSABILIDAD SOCIAL CORPORATIVA: EL CASO DE CRÉDIT AGRICOLE ESPAÑA
}

\author{
Pedro Aceituno-Aceituno, Ramiro Cea-Moure, José-Luis Casado-Sánchez y \\ Carmen Ruiz-De-Azcárate-Varela
}

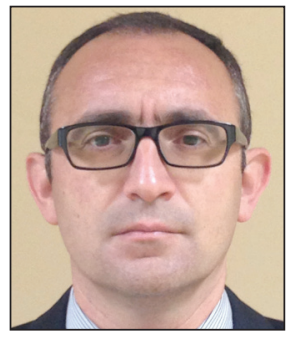

Pedro Aceituno-Aceituno es profesor de creación, gestión y organización de empresas y director de los masters en dirección económico-financiera y en auditoría de cuentas de la Universidad a Distancia de Madrid (Udima). Desde 2005 es el investigador principal de informe Innovacef de movilidad científica española. En 2003 se doctoró en ciencias económicas y empresariales por la Universidad Nacional de Educación a Distancia (UNED).

http://orcid.org/0000-0001-9034-8673

Universidad a distancia de Madrid Carretera de La Coruña, Km.38,500. Vía de servicio, n. 15 28400 Collado Villalba (Madrid), España pedro.aceituno@udima.es

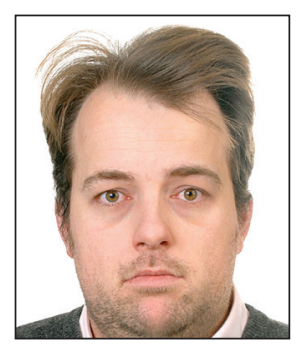

Ramiro Cea-Moure es profesor ayudante en la Universidad de Burgos y profesor tutor en la UNED. Doctor en ciencias económicas y empresariales por la Universidad Autónoma de Madrid (UAM), está acreditado como profesor ayudante doctor por la Agencia de Calidad, Acreditación y Prospectiva de la Comunidad Autónoma de Madrid (ACAP) y por la Agencia de Calidad para el Sistema Universitario de Castilla y León (Acsucyl). Ha sido becario del programa de postgrado para la Formación del Profesorado Universitario (FPU). Ha realizado una estancia de investigación en el Centre for Research into Organizational Governance.

http://orcid.org/0000-0002-2935-3016

Universidad de Burgos Hospital del Rey, s/n. 09001 Burgos, España rcea@ubu.es

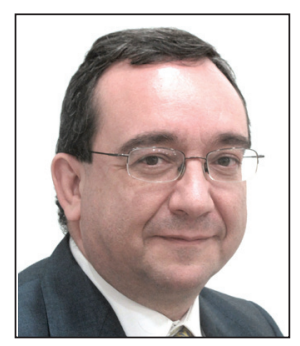

José-Luis Casado-Sánchez es profesor asociado de economía y organización de empresas en la Escuela Técnica Superior de Ingenieros (ETSI) de la Universidad Politécnica de Madrid (UPM). Auditor de cuentas inscrito en el Registro Oficial de Auditores de Cuentas (ROAC). Licenciado en ciencias económicas por la UNED y executive MBA por el IESE. Dirige empresas y proyectos de consultoría financiera. En 2012 se doctoró por la Universidad Politécnica de Madrid (UPM).

http://orcid.org/0000-0003-2804-9853

Universidad Politécnica de Madrid Ríos Rosas, 21. 28003 Madrid, España joseluis.casado@upm.es

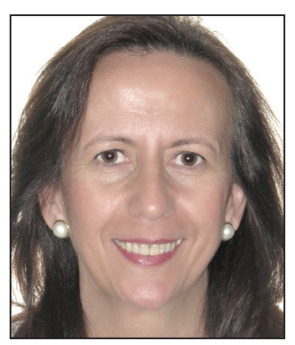

Carmen Ruiz-De-Azcárate-Varela es graduada en ciencias del trabajo y recursos humanos por la Universidad a Distancia de Madrid (Udima) desde 2012. Es miembro del Grupo de Innovación Educativa 74 de Expresión Gráfica y Cartografía de la Universidad Politécnica de Madrid y alumna del máster en educación y nuevas tecnologías de la Udima. Desde 2005 es directora comercial en Casado True \& Fair SL, consultoría estratégica y de recursos humanos.

http://orcid.org/0000-0002-8516-3671

Casado True \& Fair SL Jorge Juan, 43 tripl. 2ㅇ B. 28001 Madrid, España cruizdeazcarate@gmail.com

\section{Resumen}

Se considera la importancia de la comunicación en la implantación de la responsabilidad social corporativa (RSC) como sistema de gestión empresarial fundamental para la consecución de ventajas y el incremento de la motivación en los empleados. Se destacan las funciones a asumir por los profesionales de la información para superar las carencias en la comunicación y el desconocimiento existente en estas materias. Se ha llevado a cabo un estudio del caso de Crédit Agricole España (CAE), cuyos resultados muestran la importancia de todos estos aspectos en la implantación de la RSC. 


\title{
Palabras clave
}

Responsabilidad social corporativa, Empleados, Comunicación, Integración, Implantación, Beneficios.

Title: Communication as a key factor for implementing corporate social responsibility: the case of Crédit

Agricole España

\begin{abstract}
This paper considers the role of communication in the implementation of corporate social responsibility (CSR) as a vital management system for achieving advantages and motivation in employees. The paper also highlights the relevant functions assumed by information professionals to overcome communication deficiencies and lack of knowledge about these relatively new areas of responsibility. A case study of Crédit Agricole España (CAE) is presented, with results that highlight the importance of all the communication-related aspects of implementing CSR.
\end{abstract}

\section{Keywords}

Corporate social responsibility, Employees, Communication, Integrating, Implementation, Benefits.

Aceituno-Aceituno, Pedro; Cea-Moure, Ramiro; Casado-Sánchez, José-Luis; Ruiz-de-Azcárate-Varela, Carmen (2013).

"La comunicación como factor clave en la implantación de la responsabilidad social corporativa: el caso de Crédit Agricole España". El profesional de la información, julio-agosto, v. 22, n. 4, pp. 326-332.

http://dx.doi.org/10.3145/epi.2013.jul.08

\section{Carencias en la comunicación de la responsabilidad social corporativa: un campo novedoso para los profesionales de la información}

Las ventajas competitivas empresariales estriban cada vez en menor medida en la calidad de sus productos o servicios, por lo que las empresas optan por diferenciarse mediante activos o sistemas de gestión intangibles. Entre estos últimos destaca la responsabilidad social corporativa (RSC), que además de reportar ventajas competitivas, puede lograr otras muy importantes en términos de productividad, incremento de ventas, reducción de costes, mejora en el acceso a los mercados financieros, buena reputación de marca y facilidad en la estrategia de comunicación (Fundesa, 2009).

La RSC puede ser definida como el reconocimiento e integración en los procesos productivos, de las preocupaciones sociales y medioambientales, implantando adecuadas prácticas empresariales que satisfagan estas preocupaciones y que configuren sus relaciones con sus stakeholders o agentes interesados en la marcha de la empresa como por ejemplo los trabajadores, accionistas, socios, directivos, proveedores, clientes y sociedad en general (De-la-CuestaGonzález et al., 2002).

Pese a estas ventajas, resulta sorprendente que la RSC, no esté implantándose ampliamente. Sobre ella no existe actualmente un único estándar nacional o internacional y es complicado acceder a fuentes de información fiables, lo que hace muy difícil conocer su grado de implantación, aunque se cree que no es muy elevado (Attitudes, 2009).

En el caso español, para Mababu-Mukiur (2010) las mayores empresas siguen la tendencia internacional formalizando políticas de RSC e incluso las universidades y las escuelas de negocios incluyen esta materia en sus planes de estudio y se ha incrementado su número de horas lectivas. Todo esto unido a la proliferación de iniciativas como guías, indicadores, observatorios, premios, conferencias o talleres no encuentra reflejo en un cambio de la cultura empresarial hacia valores más responsables y éticos.

Cea-Moure (2010) analizó una muestra de las 51 entidades bancarias de la Unión Europea (UE) de mayor dimensión. De su análisis se desprende, que la política responsable más aplicada ( $80 \%$ de las entidades) es la formulación de una declaración de la alta dirección respecto a la importancia de la sostenibilidad dentro de la compañía. Ello hace pensar que la RSC no sea más que una mera declaración de intenciones.

Esta escasa concienciación también se observa en el grado deficiente de comunicación de la RSC. Las conclusiones del estudio realizado por Fontrodona y De-los-Santos (2004) entre las 500 mayores empresas españolas pusieron de manifiesto que un $20 \%$ de las compañías que tenían redactado un código de conducta, no lo habían comunicado a todos sus empleados y que el compromiso ético era menor entre los mandos intermedios y los empleados, precisamente por esta falta de comunicación.

A pesar de ello, la RSC se está implantando en las grandes empresas, debido al avance -especialmente desde el último cuarto del siglo pasado- de una nueva sensibilidad social preocupada por el poder creciente de estas organizaciones y su impacto sobre el entorno (Bueno-Campos, 2008). Es necesario incorporar a las pequeñas y medianas empresas (pymes) al discurso de la RSC (Urrilagoitia; Murillo; Lozano, 2009), dado que son el tipo de organización de la producción más abundante en cualquier economía (Fisher et al., 2009).

En estas pymes es en las que desde finales del siglo pasado, autores como Pastor (1994) han destacado la relevante labor a abordar por los especialistas en información empresarial como gestores de la información informal, muy relacionada con intangibles como la imagen, la reputación 
corporativa o la gestión del conocimiento. Recientemente Carrillo-Durán y Nuño-Moral (2010) también resaltan el inmenso y novedoso campo que las pymes constituyen para los documentalistas, dentro de su estrategia comunicativa en general y en la gestión de intangibles en particular.

Estas funciones se enlazan con competencias como la comprensión de los procesos de negocio y la cultura de la empresa, ser buen comunicador o ser formador, entre otras, que les son exigidas a los profesionales de la información en relación con las tecnologías de la información y la gestión del conocimiento (Hernández-Pérez; Rodríguez-Mateos, 2002). Dada la importancia que tiene para los resultados de la empresa la gestión coherente de estos dos últimos aspectos (Donate-Manzanares; Guadamillas-Gómez, 2008), es por lo que podría hacerse más necesaria la presencia de estos profesionales en las compañías privadas.

Los bibliotecarios-documentalistas podrían abordar estas carencias en la comunicación de la RSC anteriormente expuestas, ya que sólo con la comunicación se crea una relación fundada en valores comunes generadora de confianza y cohesión tanto para unos empleados más motivados por trabajar en una empresa responsable como para el resto de los stakeholders (Briceño; Mejías; Moreno, 2010).

Según Cabanas y Vilanova (2004), se observó una profundización en estos aspectos en la Cuarta mesa redonda del Observatorio de Comunicación Interna, organizada bajo el lema "La RSC como factor de motivación para la comunicación interna". En este evento, los departamentos de comunicación interna y RSC de 7 empresas (Vodafone, Font Vella, Hospital Clínic de Barcelona, Winterthur, Deutsche Bank, Transports Metropolitans de Barcelona y Globalia), expusieron sus experiencias acerca de cuál era el mejor modelo para implantar la RSC, decidiendo si debía ser la empresa la responsable de la comunicación y del resto de tareas para motivar e integrar a sus empleados en la realización de acciones sociales, o bien, si estas iniciativas debían proceder y ser gestionadas por los empleados, buscando el apoyo de la compañía. De las 7 empresas todas optaron por la primera opción, excepto una que trató de integrar los dos modelos.

\section{La RSC es un sistema de gestión empre- sarial con numerosas ventajas, pero de escasa implantación}

De acuerdo con estas experiencias, para tener éxito en la implantación de la RSC, ésta debe ser considerada como un objetivo estratégico a alcanzar mediante el establecimiento de un departamento, que comunique, motive e impulse tanto interna como externamente las distintas acciones a realizar, integradas todas ellas dentro de un plan con los resultados en el triple ámbito: económico, social y medioambiental.

Todo lo anterior, refleja que la RSC es un sistema de gestión empresarial con numerosas ventajas, pero de escasa implantación. Permanece en la mayoría ocasiones en la esfera de la alta dirección y escasamente llega a las pymes. A continuación se presenta un caso práctico para confirmar el relevante papel de la comunicación en la implantación de la RSC y mostrar las importantes funciones a desempeñar en esta materia por los profesionales de la información.

\section{Metodología}

Se presenta el caso de Crédit Agricole España (CAE). Con anterioridad a este trabajo, no tenía formalizadas políticas de RSC, aunque sí efectuaba acciones socialmente responsables por iniciativa de sus empleados. Ejemplo de éstas destacan los acuerdos y colaboraciones con la Fundación Seres, Adecco ETT, Fundación Prodis, Asociación Síndrome de Down, Asociación Juan XXIII o la Fundación Fundeso.

Adicionalmente, otros aspectos hacen relevante la experiencia de $C A E$ :

- Esta filial del grupo multinacional Crédit Agricole realiza su actividad en España, país donde, como se ha dicho, la implantación de la RSC es escasa, especialmente en entidades de pequeña dimensión, como CAE.

- La crisis financiera ha empeorado la imagen del sector, por lo que una estrategia de transparencia y RSC ante la sociedad y especialmente, ante los propios trabajadores, puede recuperar la confianza perdida.

- Las acciones de RSC no habían sido comunicadas a los empleados, lo que refleja las carencias en la comunicación.

- CAE es responsable de sus resultados y, por tanto, dispone de autonomía para evaluar el grado de implantación de la RSC.

Para aplicar la metodología adecuada se celebraron reuniones previas entre el equipo investigador y la dirección de recursos humanos (DRRHH) de CAE, que lidera la implantación de la RSC. Fruto de estas reuniones se estableció a través de la intranet un cuestionario de respuesta voluntaria, de acuerdo con las positivas experiencias expuestas anteriormente por Cabanas y Vilanova (2004). Dados los objetivos de este trabajo y teniendo en cuenta que las medidas de RSC efectuadas hasta el momento no habían sido comunicadas al conjunto de los empleados, el cuestionario sirvió tanto como una primera comunicación de estas materias como de diagnóstico para proporcionar las siguientes informaciones:

- Introducción (datos de clasificación): conocer las características básicas de los trabajadores de CAE que habían recibido la comunicación, para comprobar si ésta había llegado a los diferentes estratos, y hacer que el mayor número posible de empleados tomen conciencia de la importancia de implantar una estrategia responsable y de su participación en la misma. Toda la información relativa a estos datos está contenida en la tabla 1 y el gráfico 1 .

- Bloque 1 (motivación): examinar el nivel de motivación de los trabajadores de $C A E$ con respecto a los elementos establecidos con anterioridad a la realización del estudio: participación en el proceso de toma de decisiones, retribuciones y promociones, y reconocimientos. Con ello, se pretende conocer si existe una necesidad de motivación en los empleados que pudiera ser cubierta por la RSC.

- Bloque 2 (conocimiento de la RSC): para establecer un plan que integre a todos los empleados en la realización de actuaciones responsables, éstos deben poseer infor- 
mación y conocimientos en esta materia. Por ello, con este apartado se pretende estimar el grado de conocimiento de la RSC que poseen los trabajadores.

- Bloque 3 (aplicación de la RSC): con el objeto de comunicar y empezar a integrarles en la implantación de la RSC, se trata de que los trabajadores seleccionen, entre los proyectos planteados por la entidad, aquellos que les resulten más interesantes, y que demuestren su creatividad planteando nuevos proyectos en materia de RSC. Entre los proyectos a seleccionar se encuentran los siguientes:

- Identificación y educación de jóvenes emprendedores a través de Ashoka España.

- Financiación a emprendedores sociales en España.

- Patrocinio del Proyecto Prodis con la apertura de aulas universitarias que faciliten el paso del colegio a la universidad a personas discapacitadas.

- Financiación de la Fundación Síndrome de Down dedicada a la formación de personas discapacitadas y a la animación en hospitales de enfermos infantiles.

- Solicitud de servicios de catering a la Fundación Juan XXIII, formada por personas con discapacidad.

- Patrocinio del Proyecto Huerta Ecológica Milhistorias para insertar laboralmente a personas sin hogar.

- Financiar un proyecto de la Fundación RAIS, dedicada a la inclusión social.

- Participar en la Fundación Hazloposible que trata de impulsar de manera innovadora la interacción y la participación de la sociedad en causas solidarias utilizando las nuevas tecnologías.

- Trabajo voluntario en un huerto.

- Colaboración con la Fundación Seres, que pretende lograr la sensibilización social de la empresa.

- Esponsorización de la Fundación Fundeso, que organiza una cena benéfica anual.

- Aportación económica conjunta empresa-empleado para la cesta solidaria anual.

Dentro de este apartado también se busca conocer la existencia de un grupo de empleados altamente motivados en materia responsable que, a través del liderazgo del DRRHH, pudiera asumir funciones para la implantación de la RSC. A tal fin se les preguntó por su nivel de motivación en el caso de que $C A E$ aplicase este tipo de políticas responsables. Seguidamente antes de mostrar los resultados obtenidos, en la tabla 1 se exponen los datos de la ficha técnica del estudio realizado.

La RSC es el reconocimiento e integración en los procesos productivos, de las preocupaciones sociales y medioambientales, implantando adecuadas prácticas empresariales que las satisfagan

\section{Resultados y análisis}

En la tabla 1 se observa que el grado de respuesta fue elevado (47\% del total de la población) y distribuido en todos los estratos (véase gráfico 1), por lo que se consiguió un nivel adecuado de comunicación que favorecía la concienciación de los empleados para la implantación de una estrategia responsable.

En relación con el nivel de motivación de los trabajadores, los resultados expuestos en el gráfico 2 muestran que el $84,7 \%$ está de acuerdo o totalmente de acuerdo con su participación en el proceso de toma de decisiones. Sin embargo, esta proporción se reduce fuertemente en los aspectos relativos tanto al reconocimiento por aciertos y feedback por errores (58\%) como a las políticas de promociones y retribuciones $(52,7 \%)$. Por todo ello, se puede afirmar que existe una necesidad de motivación en los empleados que puede ser cubierta por la RSC.

Por lo que respecta al grado de conocimiento de los trabajadores de CAE en materia de RSC, el porcentaje no supera en ningún caso el $50 \%$, por lo que será preciso aportar documentación como fuente de información a los trabajadores, para que conozcan los conceptos propios de la RSC.

En relación con la aplicación de políticas de RSC, el proyecto más atractivo fue la colaboración con la Fundación Síndrome de Down. Esta elección puede deberse a que este proyecto es muy conocido por la sociedad española.

\begin{tabular}{|c|c|}
\hline Ámbito & Nacional \\
\hline Universo & 280 trabajadores pertenecientes a $C A E$ \\
\hline Datos de clasificación & Sexo, edad, ciudad, unidad de negocio, salario y puesto \\
\hline Muestra & $\begin{array}{l}\text { - Cuestionarios distribuidos: } 280 \\
\text { - Cuestionarios contestados: } 131 \\
\text { - Tasa de contestación: } 47 \% \\
\text { - Afijación: Proporcional } \\
\text { - Ponderación: No procede } \\
\text { - Puntos de muestreo: } 6 \text { ciudades (Madrid, Barcelona, Bilbao, La Coruña, San Sebastián y Valen- } \\
\text { cia) y } 7 \text { unidades de negocio (banco, gestora, banca privada, leasing, factoring, seguros de } \\
\text { pago, delegación de Crédit Agricole y Sociedad de valores) } \\
\text { - Procedimiento de muestreo: cuestionario online e independiente a todo el universo del estu- } \\
\text { dio con contestación aleatoria y voluntaria por parte de los trabajadores } \\
\text { - Error muestral: Para un nivel de confianza del } 95,5 \% \text { (dos sigmas), y } P=Q \text {, el error real es de } \\
\pm 2,0 \% \text { para el conjunto de la muestra y en el supuesto de muestreo aleatorio simple }\end{array}$ \\
\hline Fecha de realización & Del 30 de mayo al 20 de junio de 2011 \\
\hline
\end{tabular}

Tabla 1. Ficha técnica de la encuesta realizada a los trabajadores de CAE 
Sexo

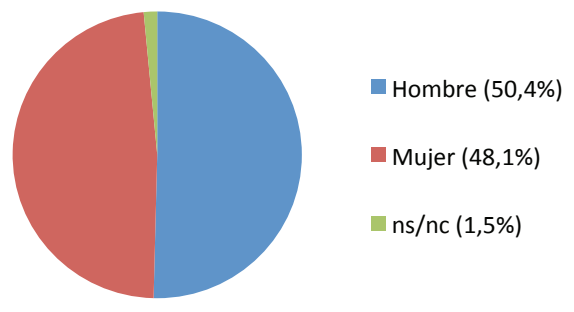

Ciudad

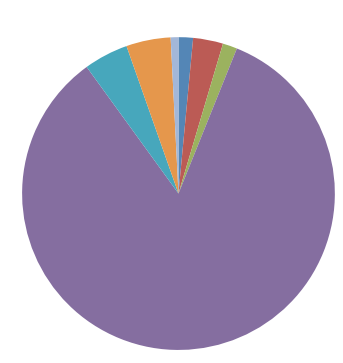

Barcelona (1,5\%)

- Bilbao $(3,1 \%)$

La Coruña $(1,5 \%)$

- Madrid (84\%)

- San Sebastián (4,6\%)

- Valencia $(4,6 \%)$

ns/nc $(0,8 \%)$

\section{Salario}

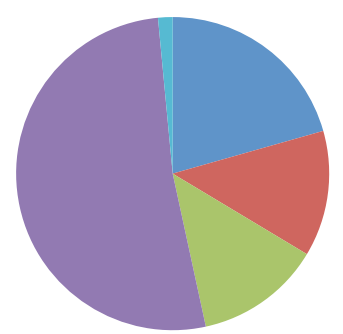

Menos de 30.000 euros $(20,6 \%)$

Entre 30.001 y 40.000 euros (13\%)

Entre 40.001 y 50.000 euros (13\%)

Más de 50.000 euros (51,9\%)

ns/nc (1,5\%)
Edad

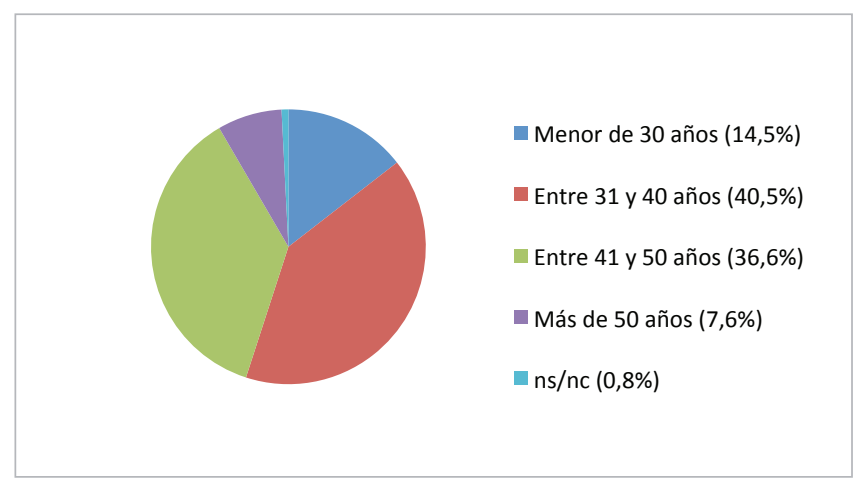

Unidad de negocio

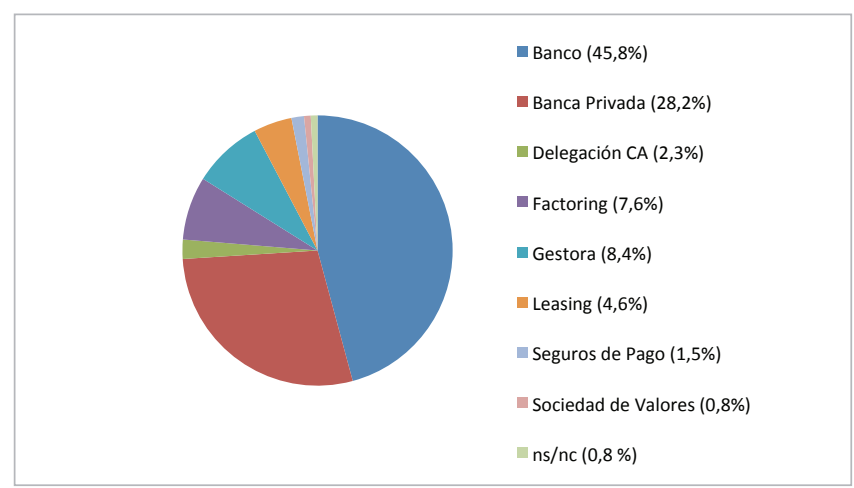

Puesto

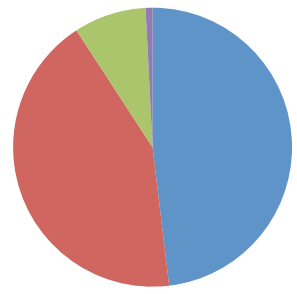

nase Operativa $(48,1 \%)$

- Mando Intermedio (42,7\%)

Dirección (8,4\%)

ns/nc $(0,8 \%)$

Gráfico 1. Porcentaje de respuestas en todas las categorías de empleados de $C A E$

En el plano creativo, los empleados propusieron proyectos de acción social (como el de Ayuda a la infancia desfavorecida, Cáritas Diocesana, Oxfam y de Apoyo a personas autistas). Parecen desconocer otras acciones que implica la RSC, y que les fueron expuestas en el Bloque 3, por lo que al igual que en el bloque anterior, resulta necesario aportar documentación en materia de RSC.

Todos estos aspectos, ponen de manifiesto una primera función a efectuar por el profesional de la información: la creación de un repositorio con documentación relativa a la RSC y datos disponibles de estas organizaciones para que cualquier persona conozca las relaciones de la empresa y pueda retomar una idea que, por ejemplo, surgió meses atrás (Pastor, 1994).

Una vez que los empleados dispongan de este conocimiento, es preciso profundizar en la comunicación interna y externa de la RSC, para lo cual, de acuerdo con Leiva-Aguilera (2010), el profesional de la información podría adoptar un perfil de community manager en una red social creada al efecto, donde realizaría las siguientes tareas adaptadas a la RSC:

- observar lo publicado en estas materias sobre la empresa y su sector;

- dirigir esta información a los departamentos y personas adecuadas;

- comunicar la posición responsable de la empresa;

- localizar y conectar a los líderes internos y externos para que colaboren en la implantación y desarrollo de la RSC.

En relación con el grado de motivación $\mathrm{x}$ de los trabajadores ante la implantación de políticas de RSC se ha utilizado la siguiente clasificación: alta $(x>7)$; media $(5<x<7)$ y baja $(0<x<4)$ :

- para el $16 \%$ de los trabajadores no aumentaría su motivación en el caso de implantarse políticas de RSC;

- un $66 \%$ experimentaría un aceptable incremento en su nivel de motivación; y

- un $18 \%$ obtendría un gran aumento. 


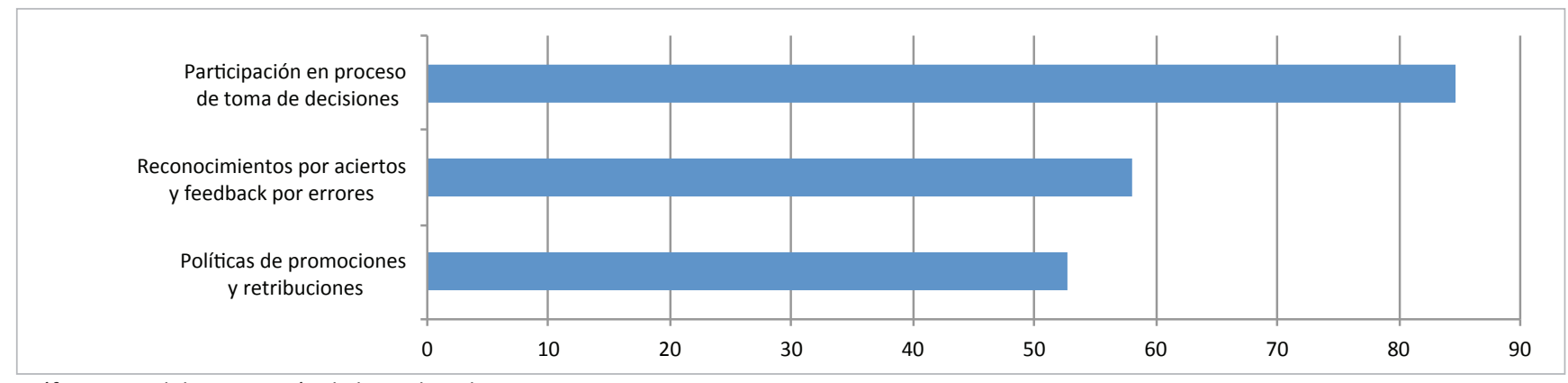

Gráfico 2. Nivel de motivación de los trabajadores

Este último porcentaje refleja que existe un grupo de trabajadores motivados para emprender proyectos responsables, que podría ayudar al DRRHH en la implantación de la RSC. Para ellos, la gestión del conocimiento a realizar por el profesional de la información debería ampliarse desde la disposición y comunicación de la información en materia de RSC hacia mecanismos alternativos que hagan que el conocimiento aflore y se comparta mediante actividades de formación, comunidades de prácticas o trabajo en equipo, por ejemplo (Soy-Aumatell, 2003).

Los empleados carecen de conocimientos en materia de RSC, que podrían ser obtenidos, aplicados y enriquecidos con la gestión del conocimiento efectuada por profesionales de la información

\section{Conclusiones}

El estudio ha servido para identificar a algunos de los empleados motivados que han ayudado al DRRHH en la implantación. Igualmente la comunicación y realización del cuestionario han permitido tener en cuenta las opiniones de los empleados en el plan 2011-2014 de implantación de la RSC.

Dentro de este plan se facilita la conciliación laboral y familiar con reducciones de jornada o una mayor flexibilidad horaria, entre otras posibilidades. También en el plano medioambiental se han establecido medidas como el ahorro de luz o el fomento del reciclaje en papel, móviles y ordenadores, en las que se cuenta con la participación de los trabajadores. Igualmente se contempla el voluntariado y durante 2012, empleados voluntarios han formado a colectivos desfavorecidos (desempleados y discapacitados) facilitando la inserción laboral de 3 de estas personas.

Estos avances en la implantación de la RSC recibieron un reconocimiento y estímulo en la Conferencia mundial de directores de recursos humanos de CA celebrada en París en octubre de 2011, siendo designado el DRRHH de CAE como líder en la alineación de medidas de RSC en todos ámbitos geográficos internacionales en los que actúe $C A$.

De acuerdo con los resultados obtenidos y los avances en la implantación de la RSC expuestos, se ha podido observar como en $C A E$, de un conjunto de acciones responsables realizadas por iniciativa de algunos empleados, pero no co- municadas y desconocidas por la mayoría de ellos, con la comunicación y el cuestionario planteado se ha evolucionado hacia el establecimiento de un plan integrador para llevar a cabo la implantación, gestión, control y mejora de la RSC.

Este relevante papel de la comunicación en la implantación de la RSC, que debe ser continuado en el tiempo, podría encontrar en la actuación de los profesionales de la información un importante apoyo. En el caso expuesto, se demuestra que los empleados carecen de muchos conocimientos en materia de RSC, que podrían ser obtenidos, aplicados y enriquecidos con la gestión del conocimiento efectuada por estos profesionales de la información. Este inmenso y novedoso campo podría suponer para ellos una salida laboral en la que aportar valor a unas entidades tan necesitadas como las pymes.

Se puede afirmar que casos como el expuesto en este trabajo demuestran que, la implantación y los avances de la RSC no hubieran sido posibles sin la comunicación de estos aspectos desde la alta dirección, integrando a todos los componentes de la empresa, bajo unos valores y actuaciones responsables.

\section{Agradecimientos}

A Victoria Magirena-Varela, directora de Recursos Humanos de CAE por su liderazgo en la proposición de actuaciones responsables y por todos los medios económicos, materiales y humanos dispuestos desde su empresa para la realización de este trabajo. Asimismo, también se agradece a la Fundación Seres, el impulso de sensibilización ejercido sobre CAE en materia de RSC. Por último, al catedrático de psicología básica Helio Carpintero-Capell por las indicaciones formuladas para la realización de este trabajo.

\section{Bibliografía}

Attitudes (2009). “Hablamos con... Víctor Saiz Pérez, analista del Observatorio de RSC".

http://www.attitudes.org/?s=saiz

Briceño, Sonia; Mejías, Iraida; Moreno, Fidel (2010) “La comunicación corporativa y la responsabilidad social empresarial (RSE)". Daena: Internacional journal of good conscience, octubre 2009-marzo 2010, v. 5, n. 1, pp. 37-46. http://www.spentamexico.org/22212/37454.html

Bueno-Campos, Eduardo (2008). Curso básico de economía de la empresa. Un enfoque de organización. Madrid: Pirámide. ISBN: 9788436819113 
Cabanas, Custodia; Vilanova, Nuria (2004). “La responsabilidad social corporativa como factor de motivación en comunicación interna". Capital humano, mayo, v. 177, pp. 70-76. http://pdfs.wke.es/1/3/6/1/pd0000011361.pdf

Carrillo-Durán, María-Victoria; Nuño-Moral, María-Victoria (2010). "La documentación en la evaluación y gestión de la imagen corporativa". El profesional de la información, 2010, marzo-abril, v. 19, n. 2, pp. 123-132.

http://www.elprofesionaldelainformacion.com/ contenidos/2010/marzo/02.pdf

http://dx.doi.org/10.3145/epi.2010.mar.02

Cea-Moure, Ramiro (2010). La responsabilidad social corporativa en la Unión Europea: análisis de sus prácticas informativas y propuesta de modelo normalizado. Tesis doctoral. Universidad Autónoma de Madrid, 2010.

De-la-Cuesta-González, Marta; Valor-Martínez, Carmen; Botija-Suiza, Manuel; Sanmartin-Serrano, Sergio (2002). La responsabilidad social corporativa: una aplicación a España. Madrid: Universidad Nacional de Educación a Distancia. ISBN: 8436247809

Donate-Manzanares, Mario-Javier; Guadamillas-Gómez, Fátima (2008). "La relación entre la postura tecnológica de la empresa y su estrategia de conocimiento. Un análisis de su efecto en los resultados". Revista europea de dirección y economía de la empresa, v. 17, n. 4, pp. 29-54.

http://dialnet.unirioja.es/servlet/articulo?codigo $=2726930$

Fisher, Kyla; Geenen, Jessica; Jurcevic, Marie; McClintock, Katya; Davis, Glynn (2009). "Applying asset-based community development as a strategy for CSR: a Canadian perspective on a win-win for stakeholders and SMEs". Business ethics: a European review, Jan., v. 18, n. 1, pp. 66-82. http://dx.doi.org/10.1111/j.1467-8608.2009.01549.x

Fontrodona, Joan; De-los-Santos, Javier (2004). Clima ético de la empresa española: grado de implantación de prácticas éticas. Documento de investigación n. 538. IESE-Universidad de Navarra.

http://ideas.repec.org/p/ebg/iesewp/d-0538.html

Fundesa - Fundación para el Desarrollo Socioeconómico del Alto Aragón (2009). Guía para la contratación de personas con discapacidad. Obra Social La Caixa.

http://goo.gl/YwHx5

Hernández-Pérez, Antonio; Rodríguez-Mateos, David (2002). "Las nuevas competencias del profesional de la información: del control de documentos a la gestión del conocimiento". En: VII Jornadas españolas de documentación: La gestión del conocimiento: retos y soluciones para los profesionales de la información, Bilbao, pp. 89-97.

http://e-archivo.uc3m.es/bitstream/10016/892/2/ competencias.pdf

Leiva-Aguilera, Javier (2010). “Comunicación en la empresa y apertura del perfil profesional de los documentalistas". El profesional de la información, marzo-abril, v. 19, n. 2, pp. 117-121.

http://eprints.rclis.org/14407/1/epi-community-manager. $p d f$

http://dx.doi.org/10.3145/epi.2010.mar.01

Mababu-Mukiur, Richard (2010). "Actitudes de los empresarios y directivos hacia la responsabilidad social corporativa". Revista de psicología del trabajo y de las organizaciones, v. 26, n. 2, pp. 101-114.

http://scielo.isciii.es/scielo.php?pid=S157659622010000200002\&script=sci_arttext

Pastor, José (1994). "Especialista en información empresarial". El profesional de la información, marzo, n. 22.

http://www.elprofesionaldelainformacion.com/ contenidos/1994/marzo/especialista_de_informacin_ empresarial.html

Soy-Aumatell, Cristina (2003). "La auditoría de la información, componente clave de la gestión estratégica de la información". El profesional de la información, julio-agosto, v. 12 , n. 4 , pp. 261-268.

http://eprints.rclis.org/15630

http://dx.doi.org/10.1076/epri.12.4.261.16889

Urrilagoitia, Lourdes; Murillo, David; Lozano, Josep (2009). RSE y pyme. Del discurso a la implementación. Una perspectiva europea. Ministerio de Industria, Turismo y Comercio, Madrid.

http://www.ipyme.org/Publicaciones/RSE-PYME.pdf

\section{Suscripción EPI sólo online}

Pensando sobre todo en los posibles suscriptores latinoamericanos, ya no es obligatorio pagar la suscripción impresa de EPI para acceder a la online.

EPI se ofrece a instituciones en suscripción "sólo online" a un precio considerablemente más reducido (96,69 +21\% IVA euros/año), puesto que en esta modalidad no hay que cubrir los gastos de imprenta ni de correo postal. 